\title{
Application of digital holography to filament size analysis
}

\author{
N V Semin ${ }^{1}$, C Poelma $^{1}$, S Drost $^{2}$ and J Westerweel $^{1}$ \\ ${ }^{1}$ Laboratory for Aero and Hydrodynamics, Faculty of Mechanical, Maritime and Materials Engineering, \\ Department of Process and Energy, Delft University of Technology, Leeghwaterstraat 21, 2628 CA Delft, \\ The Netherlands \\ 2 Teijin Aramid, Arnhem, The Netherlands \\ E-mail: N.V.Semin@tudelft.nl
}

Received 2 September 2009, in final form 12 April 2010

Published 20 May 2010

Online at stacks.iop.org/MST/21/075301

\begin{abstract}
The potential of in-line digital holography to locate and measure the size and position of filaments, i.e. thin wire-like objects, distributed throughout a thick volume has been investigated. In this paper two approaches are introduced to study filaments of varying diameter. (1) It is shown analytically and experimentally that for a gradual variation of filament diameter, one-dimensional Fraunhofer diffraction theory can be applied with an accuracy of 5\% to filaments with a diameter of 60-100 $\mu \mathrm{m}$; the $x$ and $z$ positions of filaments in a bundle can be determined with $0.1 \mathrm{~mm}$ and $0.3 \mathrm{~mm}$ accuracy respectively. (2) The out-of-focus plane approach has been modified and applied to a simple bundle of filaments for the case when their diffraction patterns can no longer be distinguished individually on the hologram. An accuracy of $20 \%$ in measuring diameter and $0.3 \mathrm{~mm}$ to detect the position is reported. Limitations as well as suggestions to further improve the technique are discussed.
\end{abstract}

Keywords: digital holography, in-line digital holography, filaments size analysis, measurement of size and location

(Some figures in this article are in colour only in the electronic version)

\section{Introduction}

Holography enables the recording of a three-dimensional (3D) optical field pattern on a two-dimensional (2D) detector [1]. A typical hologram represents an interference pattern of object and reference waves. When the hologram is simply illuminated with a coherent wave, an image of the object appears in space [2]. With the advent of electronic image sensors and powerful computers, it became possible to do the reconstruction numerically. Also, the development of image sensors (such as CCDs) enabled direct capture of holograms on a PC without involving wet chemical processing, so that nowadays acquisition and analysis of holograms can be done within a short time [3-5].

Historically one of the first practical applications of holography was in the area of particle size analysis [6]. Particle size and position measurements are important in many fields and in a variety of industries, e.g. the manufacturing of metallic powders, and the production of pharmaceuticals. Most laser- based particle analyzer techniques generally determine the distribution of the equivalent spherical particle size [7], while holography has potential to determine both the object size and location.

In this work, we apply digital holography (DH) to measure the diameter and the location of filaments in volumes of substantial depth, with the ability to distinguish individual filaments. By filaments we designate wire-like objects with varying diameter. This is of relevance, for example, in the measurement of filament thickness in the spinning process, shown schematically in figure 1 . To inspect the uniformity of the filament diameters for newly developed spinnerets, the need exists for a simple measuring technique, that is able to determine individual filament diameters (typically 40-70 $\mu \mathrm{m}$ ) in a volume of roughly $1 \mathrm{~cm}^{3}$. Moreover, a relatively large working distance of several centimeters is required because the liquid filaments are composed of a toxic acid solution. To avoid interruption of the spinning process it is desirable to perform non-intrusive inspection. The reason to apply DH 


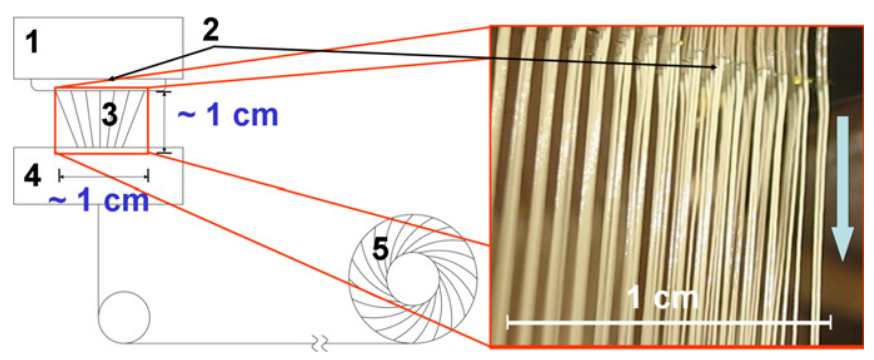

Figure 1. Left: schematic representation of the Twaron spinning process. A solution of aramid polymer in sulfuric acid (1) is pressed through a system of orifices, the spinneret (2). In this way, liquid filaments are formed with a diameter of 40-70 $\mu \mathrm{m}$ and a spacing of 1-2 mm (3). These filaments are stretched and subsequently dragged through a water bath (4), where they solidify. After passing through the water bath, the yarn is washed, dried and wound onto bobbins (5). Right: picture of liquid filaments in a small-scale spinning experiment. The arrow indicates the direction of motion.

to the problem is because the information of interest cannot be obtained with conventional imaging. There are several situations where microscopy is not suitable for imaging small objects. For instance, improving the lateral resolution of a microscope by increasing its magnification leads to quadratic decrease of the field-of-view and depth-of-field, and decreases the working distance. The depth-of-field of an optical system with $5 \mu \mathrm{m}$ resolution would be about $50 \mu \mathrm{m}$ only [8].

Our approach is somewhat based on existing methods for particle sizing. As we will show below, the particle size analysis with the help of holography has seen substantial progress in recent years. However, it strongly relies on the assumption of a spherical shape. The main challenge of this work is to overcome the poor resolution of in-line DH nowadays. In particle sizing it is less crucial because of the spherical shape of the particles. If their concentration is getting high, such that they start to obscure each other and their diffraction patterns partly overlap, the existing approaches will still work due to spherical symmetry of the particles. There is only one parameter for the shape, i.e. particle radius, and one just needs to choose carefully a proper place to measure it on a reconstructed plane where there is no overlap. This is not the case for filaments. When there are two vertical filaments close to each other they will appear as one filament of double size due to lack of spatial resolution. Thus, direct use of most methods for particle sizing to filament sizing is not possible.

Tyler et al [9] suggested the use of far-field holograms for particle size analysis. They considered the analytical process of hologram formation and reconstruction for an in-line setup for spherical particles and fibers of constant diameter. Good agreement with experiments was obtained for the diameter range of $90-160 \mu \mathrm{m}$ placed $150 \mathrm{~mm}$ from a detector. The finite aperture caused degradation of the reconstructed image quality due to edge-ringing, which could be avoided by using partly incoherent illumination during reconstruction. Vikram [10] reported another way to obtain better precision in size measurements of small particles from holograms. The reconstructed image of a hologram was viewed using a TV camera and a monitor system with an effective magnification of around 100 . This was sufficiently accurate for the size analysis of large particles, but yields significant errors for particle diameters of tens of microns. It was found that the pattern size at non-focus planes was much larger than the pattern size at the focus plane. A simple relationship between the particle size measured at a non-focus plane and the displacement from the focus plane enabled an improvement of the accuracy. A $20 \mu \mathrm{m}$ particle recorded at a distance of $6 \mathrm{~cm}$ from the camera represented a $2 \mathrm{~mm}$ dot on a monitor with a magnification of 100. At a distance of $900 \mu \mathrm{m}$ from the focus plane the particle image diameter was already $20 \mathrm{~mm}$ and the measured particle diameter was $19.87 \mu \mathrm{m}$.

Fournier et al [11] adapted numerical reconstruction of particle holograms by taking into account the diffraction envelope. This approach was aimed at improving the accuracy of the depth localization of the particles. Since the approach took into account the finite particles size, diameter extraction was also possible in principle. Denis et al [12] proposed a method to extract the mean size of particles from a digital hologram. They used a self-correlation of a particle field hologram. It was shown that the result did not depend on the distance at which the particles were located and that the slope of the correlation peak at the origin was proportional to the mean diameter of the particles. The validity of the technique was demonstrated in a cloud of $80-110 \mu \mathrm{m}$ diameter water droplets with a precision of about $10 \%$. Soulez et al [13, 14] and Gire et al [15] established the potential of in-line digital holography to locate and measure the size of particles distributed throughout a volume. They suggested an 'inverse problem' approach to improve the axial positioning accuracy, which gives particle diameters with sub-micrometer accuracy, eliminates border effects and increases the size of the measurement volume. The problem of particle detection becomes a global optimization problem, which determines the optimal set of parameters that minimizes the given penalty function. This penalty function represents the error between a model hologram and a captured hologram. Rather sparse particle distributions were used, so that individual diffraction patterns can be distinguished. An iterative algorithm finds the minimum of the penalty function and thereby determines the location and size of the particles recorded in the hologram. The validity of the technique was demonstrated for water droplets of $90 \mu \mathrm{m}$ diameter, located at a distance of $250 \mathrm{~mm}$ from a detector. The standard deviation of the diameter estimation was reported to be $0.4 \mu \mathrm{m}$.

In this paper, we apply lensless in-line $\mathrm{DH}$ to reconstruct $3 \mathrm{D}$ volumes with depth-of-field of $1-10 \mathrm{~mm}$ and a field-ofview of $10 \times 10 \mathrm{~mm}^{2}$, containing filaments of $60-80 \mu \mathrm{m}$ diameter. We demonstrate the efficacy of DH as a measuring technique. Its main advantages are that the technique is nonintrusive and applicable to measurements at relatively large distances from objects under investigation with an adequate depth of the measurement volume.

The paper is divided into three major parts. In section 2 we introduce two different approaches to measure the filament diameter from holograms: the 'inverse problem' approach and the out-of-focus approach. In section 3 experimental results are presented. Section 4 contains a summary and the conclusions, and discusses the possibilities for further improvements of the technique. 


\section{Theory}

\section{1. 'Inverse problem' approach}

The first idea of how to measure a filament diameter from its hologram is to do something similar to measuring the diameter from an image of the filament under a microscope, i.e. line by line scan through the hologram and to determine the diameter in every cross section according to a given theory that predicts the intensity distribution. Tyler et al [9] derived the analytical expression of the intensity distribution resulting from the Fraunhofer diffraction by a cylinder, aligned along the $y$ axis:

$$
\begin{aligned}
I(x) & =1-\frac{2 D}{\sqrt{\lambda z}} \cos \left(\frac{\pi x^{2}}{\lambda z}-\frac{\pi}{4}\right) \operatorname{sinc}\left(\frac{D x}{\lambda z}\right) \\
& +\frac{D^{2}}{\lambda z} \operatorname{sinc}^{2}\left(\frac{D x}{\lambda z}\right),
\end{aligned}
$$

where $D$ is the cylinder diameter, $z$ is the distance between the cylinder and the observation plane $(x, y), \lambda$ is the light wavelength. Expression (1) is valid under the condition

$$
\left\{\begin{array}{l}
\frac{\lambda}{D} \ll 1 \\
\frac{D^{2}}{\lambda z} \ll 1
\end{array}\right.
$$

According to (1), the spacing between the fringes is a function of the product $\lambda \cdot z$ only in the term $\cos \left(\frac{\pi x^{2}}{\lambda z}-\frac{\pi}{4}\right)$, whereas the envelope of the diffraction pattern $\operatorname{sinc}\left(\frac{D x}{\lambda z}\right)$ is determined by the ratio $D / z$. Consequently, the filament position can be determined from the hologram. Once this is done, one can first determine the ratio $D / z$, and then the filament diameter $D$.

This approach implies fitting of the analytical function to the experimental data. In essence, the precision of the diameter measurements is related to the number of fringes between envelope zeros, where the relative error is the inverse of the fringe count. This is true when the fringe count is not too small, say around 10 or more. One can expect that placing a filament at a distance about $30 \mathrm{~cm}$ from the CCD sensor would result in 30-40 fringes for a filament with a width of 40-60 $\mu \mathrm{m}$. Thus, a precision of approximately $2-3 \mu \mathrm{m}$ per fringe can be achieved.

This approach was first suggested by Tyler [9] for 1D wires. However, a question remains on the applicability of the approach to $2 \mathrm{D}$ objects, i.e. where the filament diameter varies along its length. To be specific, if at the object plane $(\xi, \eta)$ there is a filament with a diameter $D=D(\eta)$ that varies monotonically from $D_{0}$ to $D_{0}+\Delta D$ over a length of $\delta$ :

$$
D(\eta)= \begin{cases}D_{0}, & \eta<-\delta / 2, \\ D_{0}+\frac{\Delta D}{2}+\frac{\Delta D}{\delta} \eta, & -\delta / 2 \leqslant \eta \leqslant \delta / 2, \\ D_{0}+\Delta D, & \eta>\delta / 2,\end{cases}
$$

what would be the error when using (1) due to two dimensionality. Our goal is to theoretically estimate the allowed minimum transition distance $\delta$ and relative diameter change $\Delta D / D$ over $\delta$, so that the error in the measured diameter would be negligibly small.
If the object distribution is represented by $A(\xi, \eta)$, and it is illuminated by a collimated plane wave of amplitude $B$ and wavelength $\lambda$, then the light intensity at the recording plane $(x, y)$ at the distance $z$ from the object plane $(\xi, \eta)$ follows from the Huygens-Fresnel principle [16]:

$$
I(x, y)=B^{2}\left(1-J-J^{*}+J J^{*}\right),
$$

where

$J(x, y)=\frac{\mathrm{e}^{-\mathrm{i} \frac{\pi}{2}}}{\lambda z} \int_{-\infty}^{+\infty} \int_{-\infty}^{+\infty} \mathrm{A}(\xi, \eta) \mathrm{e}^{\mathrm{i} \frac{\pi}{\lambda z}\left[(x-\xi)^{2}+(y-\eta)^{2}\right]} \mathrm{d} \xi \mathrm{d} \eta$.

For a filament with the diameter distribution given in (3), we have

$$
A(\xi, \eta)=\operatorname{rect}\left(\frac{\xi}{D(\eta)}\right)
$$

where

$$
\operatorname{rect}(x)= \begin{cases}1, & |x| \leqslant \frac{1}{2}, \\ 0, & \text { otherwise. }\end{cases}
$$

In the far-field approximation $\left(\xi^{2} / \lambda z \ll 1\right)$ the method of stationary phase [17] can be applied to evaluate the integral (5) with the assumption $\delta^{2} / \lambda z \gg 1$ :

$$
J(x, y)=\frac{\mathrm{e}^{\mathrm{i}\left[\frac{\pi x^{2}}{\lambda z}-\frac{\pi}{2}\right]}}{\pi x} \operatorname{Im}\left(I_{1}+I_{2}+I_{3}\right),
$$

where $\mathrm{Im}$ is the imaginary part of a complex variable, and

$$
\begin{gathered}
I_{1} \approx \mathrm{e}^{\mathrm{i}\left[\frac{\pi\left(D_{0^{+}} \frac{\Delta D}{\lambda z}\right) x}{\lambda z}+\frac{\pi}{4}\right]} \mathrm{e}^{\mathrm{i} \frac{\pi}{2 \lambda z} \frac{\Delta D}{\delta} x^{2}}, \\
I_{2} \approx\left(\frac{\delta}{\sqrt{\lambda z}}\right)^{-1} \mathrm{e}^{\mathrm{i}\left[\frac{\pi D_{0} x}{\lambda z}+\frac{\pi \delta^{2}}{4 \lambda z}\right]}, \\
I_{3} \approx\left(\frac{\delta}{\sqrt{\lambda z}}\right)^{-1} \mathrm{e}^{\mathrm{i}\left[\frac{\pi\left(D_{0}+\Delta D\right) x}{\lambda z}+\frac{\pi \delta^{2}}{4 \lambda z}\right]} .
\end{gathered}
$$

The integral $I_{1}$ is a product of two terms. The first one,

$$
\mathrm{e}^{\mathrm{i}\left[\frac{\pi\left(D_{0}+\frac{\Delta D}{2} x\right)}{\lambda z}+\frac{\pi}{4}\right]},
$$

is the exact solution for a $1 \mathrm{D}$ filament of diameter $D_{0}+\frac{\Delta D}{2}$ [9]. The second multiplier of the integral $I_{1}$,

$$
\mathrm{e}^{\mathrm{i} \frac{\pi}{2 \lambda z} \frac{\Delta D}{\delta} x^{2}}
$$

as well as the integrals $I_{2}$ and $I_{3}$ account for the effect of filament diameter variation. If we require the phase of (13) to be small, i.e. $\frac{\pi}{2 \lambda z} \frac{\Delta D}{\delta} x^{2} \ll \pi$, at least over a distance of the diffraction envelope $x \approx \frac{\lambda z}{D}$, then (13) can be neglected in (9). Also, the contribution of terms (10) and (11) to (8) in comparison to (9) is of order $\left(\frac{\delta}{\sqrt{\lambda z}}\right)^{-1}$ if we increase the transition distance $\frac{\delta}{\sqrt{\lambda z}} \rightarrow \infty$. Thus, the diffraction pattern of a filament with a variable width at $y=0$ is represented by that of an infinitely long 1D filament under the condition

$$
\left\{\begin{array} { l } 
{ \frac { \delta ^ { 2 } } { \lambda z } \gg 1 } \\
{ \frac { \pi } { 2 \lambda z } \frac { \Delta D } { \delta } x ^ { 2 } \ll \pi }
\end{array} \quad \text { or equivalently } \quad \left\{\begin{array}{l}
\delta \gg \delta^{*} \\
\frac{\Delta D}{D} \ll \frac{\delta}{\delta^{*}} \frac{D}{\delta^{*}},
\end{array}\right.\right.
$$



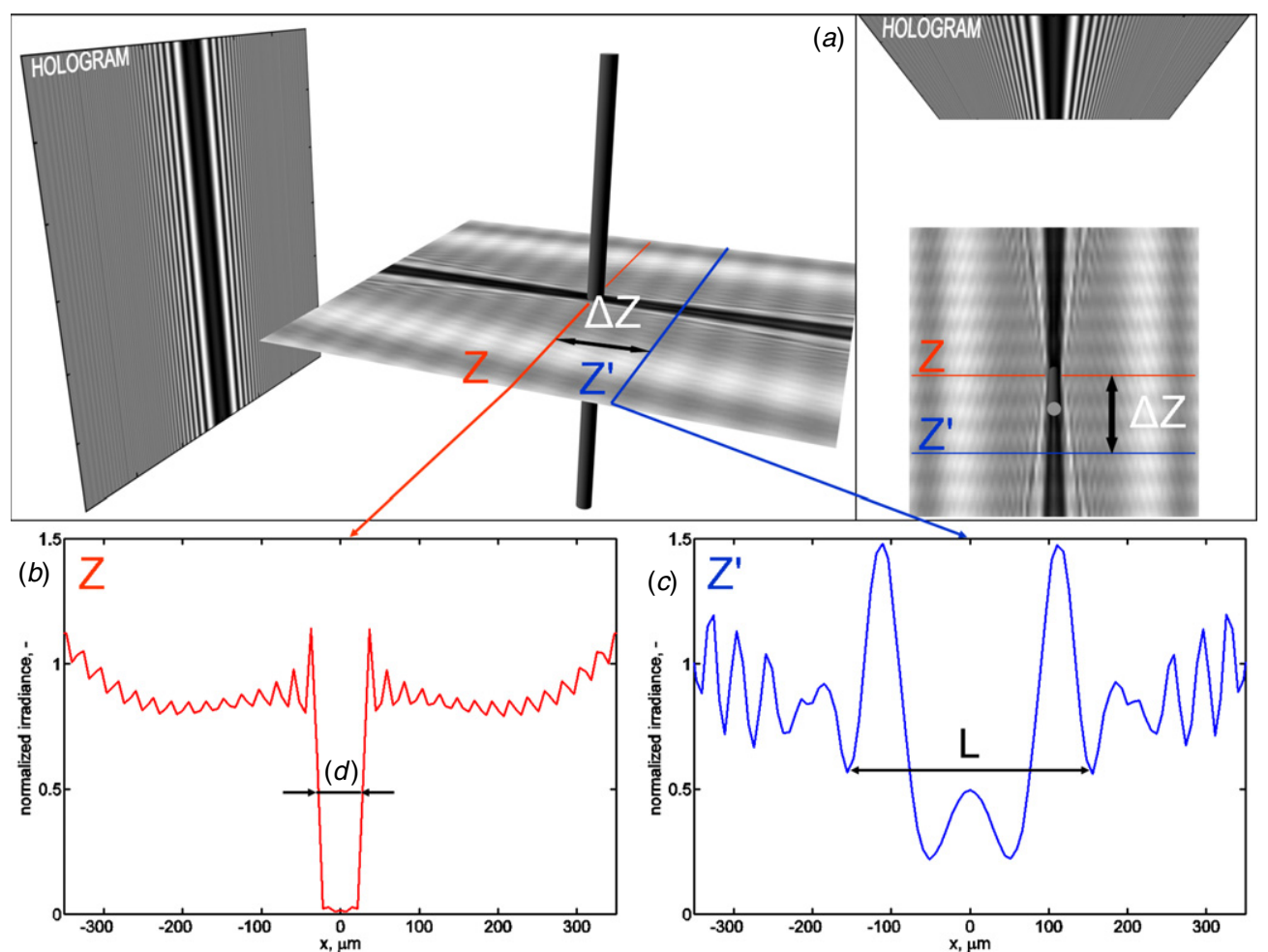

Figure 2. Illustration of the out-of-focus approach working principle: $(a)$ the side view and top view of the reconstruction process; (b) irradiance distribution at the plane $z ;(c)$ irradiance distribution at the plane $z^{\prime}=z+\Delta z$.

where $\delta^{*} \equiv \sqrt{\lambda z}$. Relationship (14) means that the distance over which a substantial change in diameter takes place should be larger than some characteristic length scale $\delta^{*}$, and the relative change in the diameter $\Delta D / D$ should be sufficiently small. In this case correction terms for two dimensionality (10), (11) and (13) can be neglected.

In our experiments, $\lambda=0.634 \mu \mathrm{m}, z=150 \mathrm{~cm}$ and $D \sim 100 \mu \mathrm{m}$. For these values $\delta^{*} \sim 300 \mu \mathrm{m}$; thus, the maximum allowed relative change of diameter $\Delta D / D$ should not exceed $D / \delta^{*} \sim 30 \%$.

\subsection{Out-of-focus plane approach}

Figure 2 explains the working principle of the out-of-focus plane approach to measure the filament diameter from its hologram. After a hologram is captured on a CCD sensor, digital reconstruction of the field can be done on a PC. Figure 2 shows the side view and top view of the process. Light rays emanating from the hologram form a focus at the plane $z$. The field pattern in this plane (figure $2(b)$ ) corresponds to the original object, which was used to record the hologram. One can measure the filament diameter $D$ from the pattern as illustrated in figure $2(b)$. However, it is difficult to obtain a satisfactory result, since the resolution of the measurements is limited by the pixel dimensions of the CCD sensor. A $60 \mu \mathrm{m}$ diameter filament would occupy about 10 pixels in the focal plane, and due to edge ringing in the reconstruction the uncertainty would be at best $2-3$ pixels, or $20-30 \%$. For some applications this may be too coarse.
The further propagation of light rays shows a certain diverging pattern with clear maxima and minima. At the outof-focus plane $z^{\prime}=z+\Delta z$ the width between first symmetric minima $L$ (which we could refer as the out-of-focus diameter) is much larger than $D$, i.e. about $300 \mu \mathrm{m}$ or 30 pixels (figure 2(c)). Consequently, a 2-3 pixel uncertainty in measuring $L$ is only $5-10 \%$. If we can try to relate the displacement of the out-of-focus plane from the image plane $\Delta z$, measured distance between symmetric minima at the outof-focus plane $L$ with the diameter of the filament $D$, then the latter can also be determined with a better accuracy. It was done first by Vikram [10] for spherical particles, whereas here we present a derivation for the case of a cylinder.

The Huygens-Fresnel principle can again be applied to determine the reconstructed field in a plane $(u, v)$ at a distance $z^{\prime}$ from the hologram. If a plane wave of amplitude $C$ at the same wavelength is used to illuminate the hologram normally, the field is

$\Psi(u, v)=-\frac{\mathrm{i} C}{\lambda z^{\prime}} \mathrm{e}^{\mathrm{i} k z^{\prime}} \iint_{-\infty}^{+\infty} I(x, y) \mathrm{e}^{\mathrm{i} k \frac{(x-u)^{2}+(y-v)^{2}}{2 z^{\prime}}} \mathrm{d} x \mathrm{~d} y$.

Our goal is to determine the field at a slightly different plane $z^{\prime}=z+\Delta z$, where $\Delta z$ is the distance from the primary focus plane. If the object distribution at the object plane is one dimensional, then substituting (4) in (15) in the far-field condition $\xi^{2} / \lambda z \ll 1$ and applying the method of stationary phase to evaluate the integral, leads to the intensity at the 


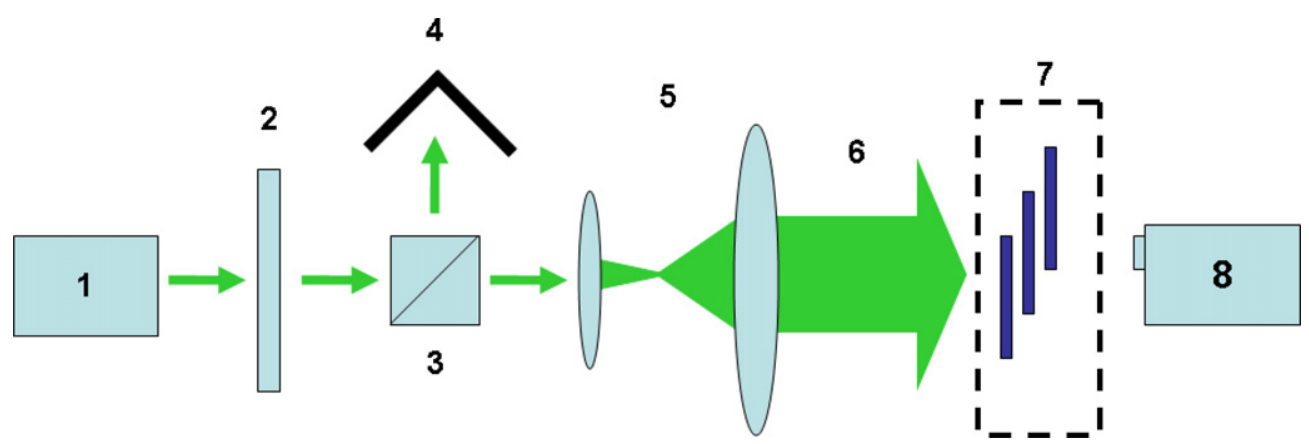

Figure 3. Schematic of the experimental setup: (1) laser; (2) half-wave plate; (3) polarized beam splitter; (4) beam dump; (5) lenses; (6) collimated laser beam; (7) specimen; (8) CCD camera.

observation plane $(u, v)$

$$
\begin{gathered}
I(u, v)=\left.\Psi(u, v)\right|^{2} \propto \mid 1-\frac{\mathrm{e}^{\mathrm{i}\left[\frac{k u^{2}}{2 \Delta z}-\frac{\pi}{4} \operatorname{sign} \Delta z\right]}}{\sqrt{\lambda \Delta z}} \tilde{A}^{*}\left(-\frac{u}{\lambda \Delta z}\right) \\
-\frac{\mathrm{e}^{\mathrm{i}\left[\frac{\mathrm{i}_{k u^{2}}}{2\left(z+z^{\prime}\right)}-\frac{\pi}{4}\right]}}{\sqrt{\lambda\left(z+z^{\prime}\right)}} \tilde{A}\left(\frac{u}{\lambda\left(z+z^{\prime}\right)}\right)+\left.\frac{\left|\tilde{A}\left(\frac{x}{\lambda z}\right)\right|^{2}}{\lambda z}\right|^{2} .
\end{gathered}
$$

For $|\Delta z| \ll z$ and $z \gg D^{2} / \lambda$, the last two terms of (16) can be neglected and result in

$$
I(u, v) \propto\left|1-\frac{\mathrm{e}^{\mathrm{i}\left[\frac{k u^{2}}{2 \Delta z}-\frac{\pi}{4} \operatorname{sign} \Delta z\right]}}{\sqrt{\lambda \Delta z}} \tilde{A}^{*}\left(-\frac{u}{\lambda \Delta z}\right)\right|^{2} .
$$

For a 1D object, like a thin filament with

$$
A(\xi)=\operatorname{rect}\left(\frac{\xi}{D}\right)
$$

it is found that

$$
\tilde{A}^{*}\left(-\frac{u}{\lambda \Delta z}\right)=D \operatorname{sinc}\left(\frac{u D}{\lambda \Delta z}\right) .
$$

This results in

$$
\begin{gathered}
I(u, v) \propto 1-\frac{2 D}{\sqrt{\lambda \Delta z}} \cos \left(\frac{k u^{2}}{2 \Delta z}-\frac{\pi}{4} \operatorname{sign} \Delta z\right) \\
\times \operatorname{sinc}\left(\frac{D u}{\lambda \Delta z}\right)+\frac{D^{2}}{\lambda \Delta z} \operatorname{sinc}^{2}\left(\frac{D u}{\lambda \Delta z}\right) .
\end{gathered}
$$

It is no coincidence that (20) is very similar to (1). The reason for this is that when reconstructing the hologram, we observe at the focal plane a real image of the original object, i.e. a 1D filament. Further propagation of light waves is described again by the Huygens-Fresnel principle. Consequently, under the far-field approximation at a non-image plane we observe Fraunhofer diffraction from a real image of a filament.

Our basic goal is to measure at large distances, compared to the filament diameter, i.e. $u \gg D$. With this assumption and $\Delta z \geqslant D^{2} / \lambda$, the second term on the rhs of (20) can be neglected due to the high-frequency term $\cos \left[\frac{\mathrm{i} \mathrm{u}^{2}}{2 \Delta z}\right]$, that is beyond the resolution of the CCD sensor.

Thus, under the physical conditions stated above, we have as a first-order approximation

$$
I(u, v) \propto 1+\frac{D^{2}}{\lambda \Delta z} \operatorname{sinc}^{2}\left(\frac{D u}{\lambda \Delta z}\right) .
$$

Detecting the maxima or minima of the pattern at out-of-focus planes allows the calculation of the diameter of the filament at the image plane:

$$
D=2 k \frac{\lambda \Delta z}{L_{k}}, \quad k=0,1,2, \ldots,
$$

where $L_{k}$ is the measured distance between the corresponding minima of $k$ orders at the out-of-focus plane.

\section{Experimental details}

\subsection{Experimental setup}

The experimental setup is presented in figure 3. An essential requirement is a collimated uniform laser beam profile. For this purpose, a pair of good quality lenses is necessary ( 5 in figure 3 ) to expand and collimate the beam. The combination of a half-wave plate and a polarized beam splitter ( 2 and 3 in figure 3) was used to manually control the intensity. This achieves an optimal signal-to-noise ratio and optimal use of the dynamic range of the CCD sensor.

\subsection{Validation of the $2 D$ filament approach}

To validate the theoretical results from section 2.1 a glass needle of varying diameter was used (figure $4(a)$ ). A reference measurement of the needle tip was carried out with the help of a profile projector. It turned out that over a length of about $10 \mathrm{~mm}$ the needle tip diameter varies monotonically from about $20 \mu \mathrm{m}$ up to $270 \mu \mathrm{m}$, which was measured with an uncertainty of the order of $2.5 \mu \mathrm{m}$ (1 in figure $4(d)$ ). The needle was placed about $15 \mathrm{~cm}$ from the CCD sensor. The value of $\delta^{*}$ was $300 \mu \mathrm{m}$ or 46 pixels. According to the theory, it would be enough to examine only cross sections located 46 pixels from each other to determine the needle size. To improve the accuracy the results are averaged over seven lines.

The processing was as follows. First, the hologram of the needle was captured. In order to remove noise, a background image without the specimen was recorded and then subtracted from original data (figure $4(b)$ ). Then the hologram was digitally reconstructed (figure $4(c)$ ) at a distance of $15 \mathrm{~cm}$ and binarized with a threshold of $10 \%$ to determine the $y$ coordinate of the needle and, with the classical approach to 


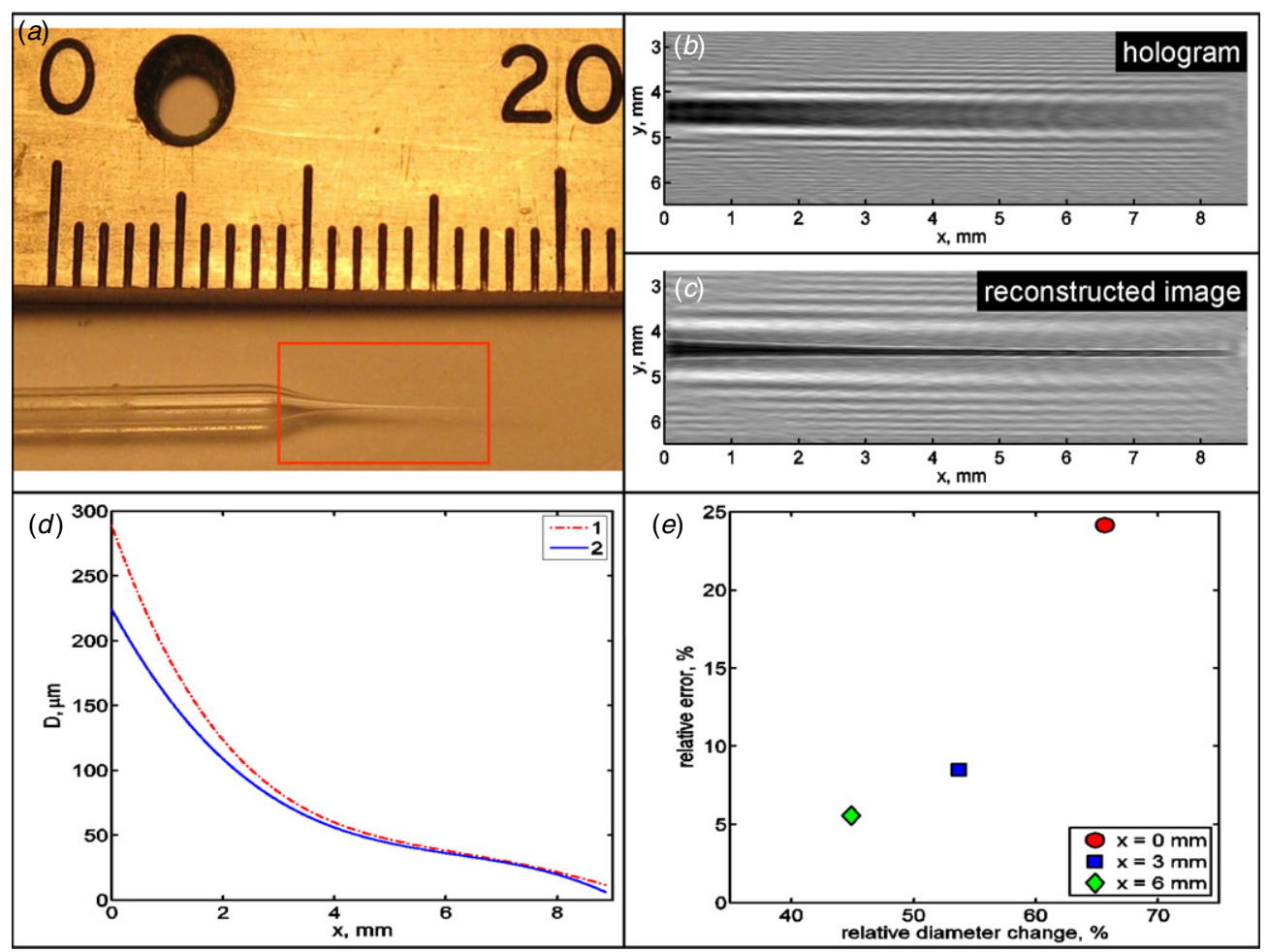

Figure 4. 'Inverse problem' approach results for the glass needle: $(a)$ a glass needle that was used to demonstrate the validity of the technique; $(b)$ a hologram of the needle after background subtraction; $(c)$ a reconstructed image at a distance $z=15 \mathrm{~cm} ;(d)$ dashed-dotted line is the direct estimate for the needle diameter in $(a)$; the solid line is the final approximation of the needle diameter after the fitting procedure; $(e)$ relative error of diameter measurements $\left(D-D_{0}\right) / D_{0}$ versus relative diameter change $\Delta D_{0} / D_{0}$ every $\delta=3 \mathrm{~mm}$.

analyze holograms [16], to get a first estimate for the needle diameter.

The next step is to fit the analytical formula (1), using a penalty function, to the experimental data at selected cross sections. The initial guess for the location of the needle was $150 \mathrm{~mm}$, while after fitting it was refined to $142.5 \mathrm{~mm}$. In figure $4(d)$ two results are plotted: the (red) dashed-dotted line corresponds to the direct needle diameter measurements with the help of the profile projector; the (blue) solid line is the final approximation of the needle diameter after the fitting procedure.

Finally, figure 4(e) represents the relative error of the diameter measurements $\left(D-D_{0}\right) / D_{0}$ versus relative diameter change $\Delta D_{0} / D_{0}$, where $D_{0}$ is the reference measurement of the diameter with the profile projector (red dashed-dotted line in figure $4(d)$ ), $D$ is the diameter value obtained with the fitting approach (blue solid line in figure $4(d)$ ). The diameter change $\Delta D$ is taken every $\delta=3 \mathrm{~mm}$ to satisfy the requirement (14): $\delta \gg \delta^{*}=0.3 \mathrm{~mm}$. In the interval $0 \leqslant x \leqslant 3 \mathrm{~mm}$ the relative diameter changes almost $70 \%$ and the error is $24 \%$. In the interval $6 \leqslant x \leqslant 9 \mathrm{~mm}$ the relative diameter change is not so large, i.e. $45 \%$, and the relative error drops to less than $5 \%$. This is in good agreement with our estimate in section 2.1.

\subsection{Test object with three filaments}

Subsequently we applied the method to multiple filaments. In the right picture of figure 1 the liquid filaments emerge from 50 holes, organized in 5 rows by 10 orifices in each. In each row orifices have a $1 \mathrm{~mm}$ spacing, with the rows being located 1-3 mm from each other. Consequently, the depth of field for the volume of interest is about $4-10 \mathrm{~mm}$. To model this case in the laboratory we used human hair as a substitute for liquid filaments. Human hair has a diameter of $60-100 \mu \mathrm{m}$, which is comparable to the filament diameter size range of 40-70 $\mu \mathrm{m}$. The hairs were attached to a frame in such a way that it could rotate approximately around the middle hair. In two data sets the hairs were placed 3 and $5 \mathrm{~mm}$ from each other (figure 5). The angle of rotation varied from $0^{\circ}$ (a plane parallel to the CCD sensor) to $80^{\circ}$ (a plane almost perpendicular to the CCD sensor).

\section{4. 'Inverse problem' approach}

In figure 6(a) a post-processed hologram for the case of three filaments placed about $18 \mathrm{~cm}$ from the CCD sensor is presented. The indices 1-3 identify the filaments from left to right. The frame with the filaments is rotated $45^{\circ}$ around the $z$ axis. The goal is to determine the diameter and position. In figure $6(b)$ the comparison between the experimental data and the final fit is shown for a line at $y=4 \mathrm{~mm}$. The two plots do not seem to coincide completely. The point is that the scaling in $y$ does not play any role at all. The data that are being fitted are in fact the coordinates where the diffraction envelope intersects $y=1$. Information on the diameter is encoded in the number of fringes between the middle of the diffraction pattern and the first zero of the envelope function. The difference in fringes scaling between experimental data 


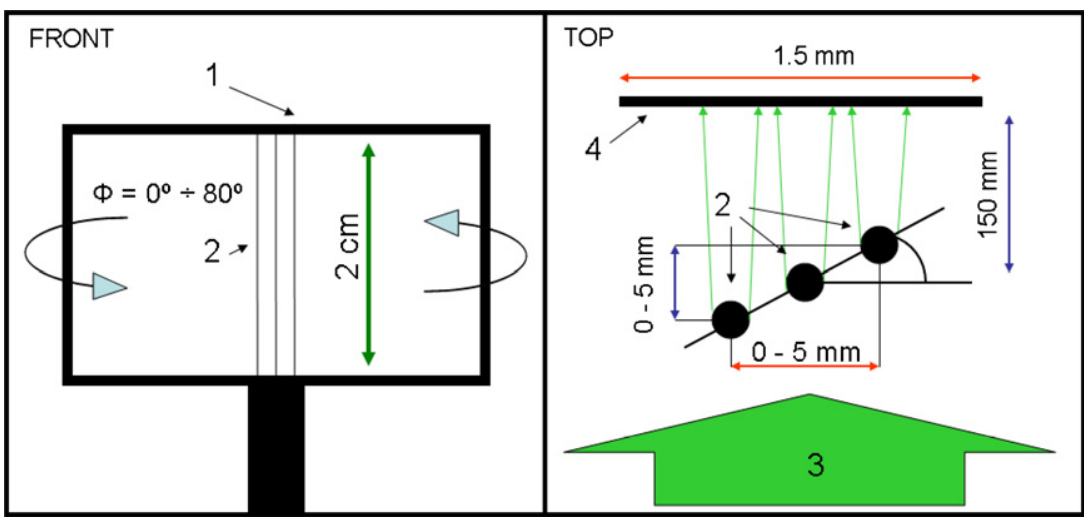

Figure 5. Test target with three filaments (human hair). Front: (1) frame with three hairs; (2) hair. Top: (2) hair; (3) laser beam; (4) CCD sensor.

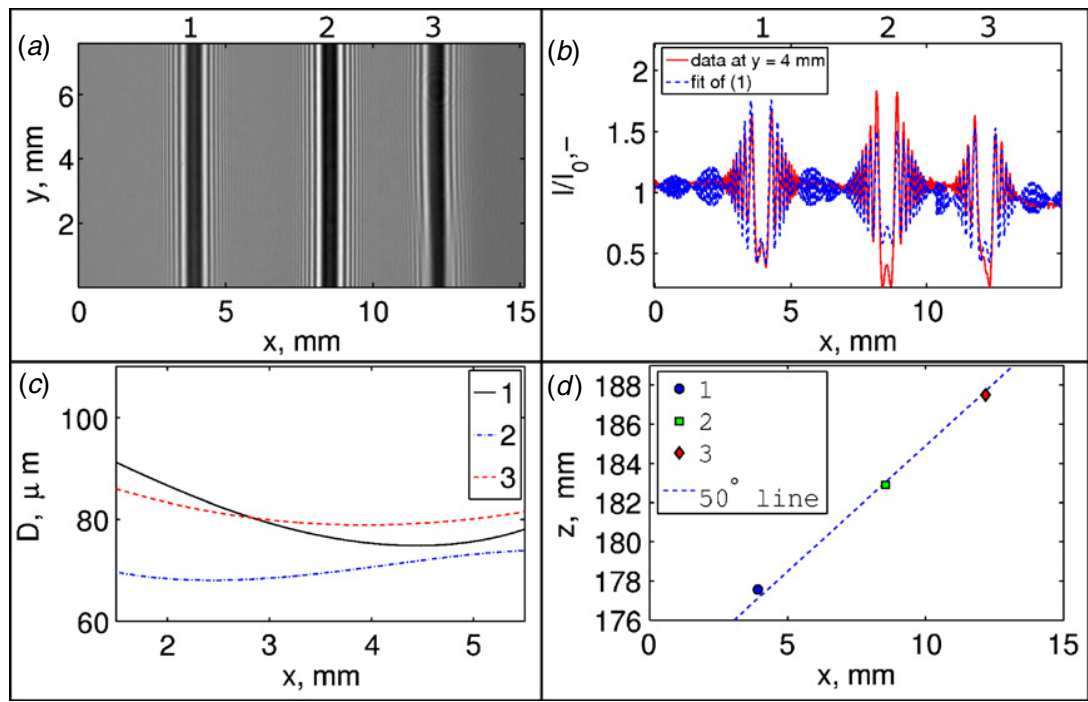

Figure 6. 'Inverse problem' approach results for a small bundle of filaments: $(a)$ a post-processed hologram for the case of three filaments placed about $18 \mathrm{~cm}$ from the CCD sensor; the indices 1-3 identify the filaments; $(b)$ a comparison between the experimental data and the final fit at $y=4 \mathrm{~mm} ;(c)$ filament diameter as a function of the $y$ coordinate; $(d)$ filaments' $x$ and $z$ coordinates.

and the fitting curve for the second filament indicates that the filament was undergoing rapid changes in diameter around this location.

Fitting enables us to extract the filament diameter and location, presented in figures $6(c)$ and $(d)$ respectively. From figure $6(d)$ it can be inferred that the angle between the frame with filaments and the $x$ axis was roughly $50^{\circ}$.

\subsection{Limitations of the 'inverse problem' approach}

The main limitation of the approach is the assumption that individual diffraction patterns can be recognized in the hologram, or, in other words, that the total irradiance is the sum of individual irradiances. This is only the case when the filaments are not too close to each other. The minimum allowable distance is determined by the size $L$ of the diffraction envelope, that is, approximately by

$$
L=\frac{\lambda z}{d} \text {. }
$$

Assuming $\lambda=0.632 \mu \mathrm{m}, z=15 \mathrm{~cm}, d=50 \mu \mathrm{m}$, gives $L=2 \mathrm{~mm}$. For the results presented in figure 6 the spacing between the filaments is about $5 \mathrm{~mm}$.

\subsection{Out-of-focus approach}

To overcome the limitation of the 'inverse problem' approach we consider the out-of-focus plane approach. Figure $7(a)$ is a hologram of three filaments, placed initially $3 \mathrm{~mm}$ from each other. The frame with the filaments was rotated about $60^{\circ}$ around the $z$ axis. Thus, effectively, the observed spacing in the hologram is about $1.7 \mathrm{~mm}$. Figure $7(b)$ shows a cross section of the hologram for $y=0.8 \mathrm{~mm}$. It is clear that for this case individual diffraction patterns interfere significantly, and the 'inverse problem' approach can no longer be used.

Figure $7(c)$ shows a cross section of 200 reconstructed planes, located between $175 \mathrm{~mm}$ and $230 \mathrm{~mm}$ from the hologram in figure 7(a). The position of the filaments can be determined at places where light rays form a focus (circles in figure $7(a)$ ). The indices $1-3$ in figure $7(c)$ identify the filaments from left to right in figure $7(a)$. The dashed line 


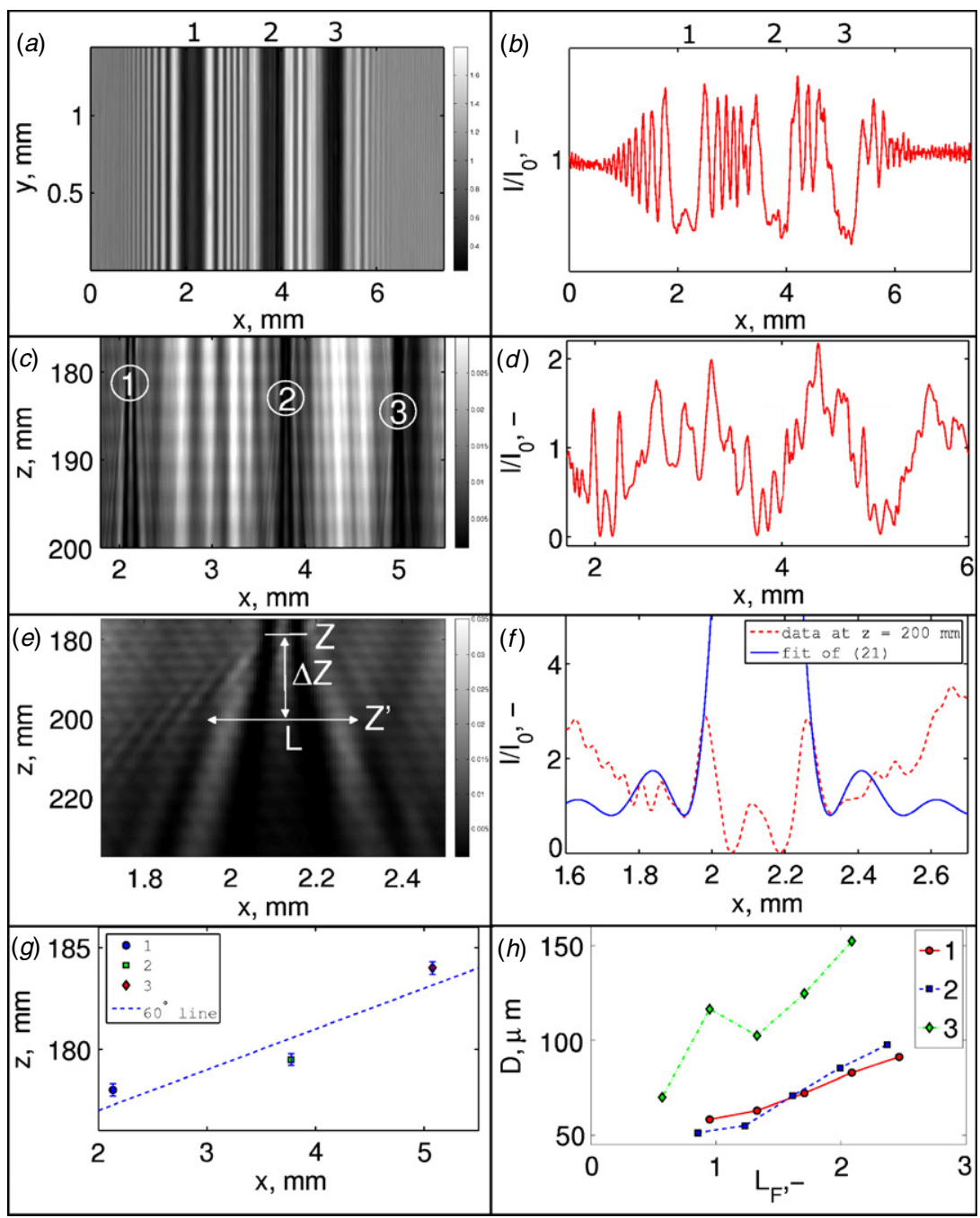

Figure 7. Out-of-focus plane approach results: (a) hologram of three hairs, placed initially $3 \mathrm{~mm}$ from each other; the indices $1-3$ identify the filaments. The frame with the filaments is rotated about $60^{\circ}$ around the $z$ axis. (b) Cross section of the hologram for $y=0.8 \mathrm{~mm}$; $(c)$ cross section of 200 reconstructed planes, located between $175 \mathrm{~mm}$ and $230 \mathrm{~mm}$ from the hologram in $(a)$; $(d)$ irradiance distribution at $z=200 \mathrm{~mm} ;(e)$ magnification of area 1 in $(c) ;(f)$ magnification of the area in $(d)$ for $1.6 \leqslant x \leqslant 2.6 \mathrm{~mm} ;(g)$ the $x$ and $z$ position of the filaments; $(h)$ diameter of the filaments versus distance in far fields $\left(L_{F}\right)$ from the focal plane.

in figure $7(d)$ is an irradiance distribution at $z=200 \mathrm{~mm}$ in figure 7(c). Rectangles indicate parts of the curve described by (21). Figure 7(e) shows a magnified part of figure 7(c) near the location of filament 1 . To determine the filament diameter, we first find its $z$ position ( $z$ in figure $7(c)$ ). This can be done with a precision of $0.3 \mathrm{~mm}$. After that at an out-of-focus plane that is located $\Delta z=20 \mathrm{~mm}$ or roughly one far-field distance from the image plane $\left(z^{\prime}\right.$ in figure $7(e)$ ), the width $L$ between first minima of the irradiance plot is measured. Once this is done, we can calculate the diameter of the filament $D$ at the focus plane $z$ according to (22). Figure $7(f)$ is a magnified section of figure $7(d)$ within the area located between $1.6 \leqslant x \leqslant 2.6 \mathrm{~mm}$. The solid line in figure $7(f)$ is $(21)$ plotted for the obtained values of $\Delta z$ and $D$. The same procedure was repeated to determine the diameters of filaments 2 and 3 respectively. Figure $7(g)$ shows the extracted location of the filaments with an uncertainty of $0.3 \mathrm{~mm}$. Finally, in figure $7(h)$ the diameter of the filaments measured at different positions from the focal plane is presented. The distance is normalized with the far- field distance $L_{F}$ for a filament of $100 \mu \mathrm{m}$ diameter, given by

$$
L_{F}=D^{2} / \lambda
$$

which is about $15 \mathrm{~mm}$ for the present case.

There is a weak dependence of measured diameters on the distance from the focal plane for $1-2 \times L_{F}$. With increasing distance the error increases to $30 \%$. This can be explained because the further we go from the focal plane the more crude the approximation $\Delta z \ll z$ becomes.

Although there is clearly an error of $10-15 \%$ in the measured diameter of filaments, with decreasing $D$, the error also decreases, since the conditions leading to (21) become more favorable. We can expect that for filaments of 40-60 $\mu \mathrm{m}$ in diameter the error can be reduced to $5 \%$.

\subsection{Limitations}

The theory is in principle one dimensional. However, the same arguments can be applied in this case to show that under 
assumptions (14), the error due to two dimensionality will be insignificant.

Secondly, the approximation (21) is valid if the observation plane is several times the distance $L_{F}$ away from the focal plane. On the other hand, the intensity decreases as $\Delta z$ increases, i.e. the signal-to-noise ratio gets worse. Preferably, $\Delta z$ should be as small as possible. To satisfy both conditions, $\Delta z$ would preferably be $\Delta z \sim L_{F}$.

Meanwhile, $\Delta z$ should remain much smaller than $z$. For $D=100 \mu \mathrm{m}$ we already have $L_{F}=15 \mathrm{~mm}$. Consequently, the smaller the filament the better the approach will work provided that the Fraunhofer approximation remains valid.

\section{Discussion and conclusion}

A study was done on the application of digital holography to micrometer filament size analysis in a volume with a large depth. Two different approaches have been proposed to extract the dimension and position of filaments that occur in a bundle.

It has been shown analytically and experimentally that for gradual variations of filament diameter over length $(\Delta D /$ $D<30 \%$ ), one-dimensional Fraunhofer diffraction theory can be applied, which yields results with an accuracy of $5 \%$ for filaments with a diameter of 60-100 $\mu \mathrm{m}$. The in-plane $(x)$ and out-of-plane $(z)$ positions of filaments in a simple bundle can be determined with a precision of $0.1 \mathrm{~mm}$ and $0.3 \mathrm{~mm}$ respectively.

The out-of-focus plane approach has been modified and applied to a simple bundle of filaments for the case when their diffraction patterns can no longer be distinguished separately on the hologram. An accuracy of $20 \%$ in measuring the diameter and of $0.3 \mathrm{~mm}$ to detect the position has been obtained.

We suggest two ways to further improve the technique. First, to utilize two or more cameras to capture holograms of a bundle of filaments from different views. This will help in improving the measurements of the position of the filaments [8]. Second is to premagnify the field before recording the hologram [18]. This will increase effective resolution of the system. As a result, reconstruction of the holograms can be done with better accuracy and the out-of-focus approach will perform better as well.

In order to apply the method to the bundle of filaments shown in figure 1 , one can capture the hologram of the bundle at a slight angle with the $z$ axis, so that the filaments are not blocked by each other. The big bundle of filaments can be considered as a row comprising several small bundles with five filaments in a line. Then each small bundle can be investigated further with the approaches suggested in this paper.

\section{Acknowledgments}

The authors wish to express their gratitude to T A Ooms, W D Koek, C Fournier and J Coupland for valuable discussion on the topic. Support by E Overmars and R Delfos in organizing experiments is gratefully acknowledged. We appreciate the comments and suggestions of the referees which significantly helped to improve the quality of the paper. NS would like to personally thank R Terlekchi for inspiring him in studying holography a long time ago.

\section{References}

[1] Collier R J, Burckhardt C B and Lin L H 1971 Optical Holography (New York: Academic)

[2] Gabor D and Goss W 1966 Interference microscope with total wavefront reconstruction J. Opt. Soc. Am. 56 849-58

[3] Goodman J and Lawrence R 1967 Digital image formation from electronically detected holograms Appl. Phys. Lett. 1177

[4] Kronrod M, Merzlyakov N and Yaroslavskii L 1998 Reconstruction of a hologram with a computer SPIE Milestone Series 144 645-6

[5] Schnars U and Juptner W P O 2002 Digital recording and numerical reconstruction of holograms Meas. Sci. Technol. 13 85-101

[6] Thompson B J, Ward J H and Zinky W R 1967 Application of hologram techniques for particle size analysis Appl. Opt. $6519-26$

[7] Black D L, McQuay M Q and Bonin M P 1996 Laser-based techniques for particle-size measurement: a review of sizing methods and their industrial applications Prog. Energy Combust. 22 267-306

[8] Sheng J, Malkiel E and Katz J 2006 Digital holographic microscope for measuring three-dimensional particle distributions and motions Appl. Opt. 45 3893-901

[9] Tyler G and Thompson B 1976 Fraunhofer holography applied to particle size analysis a reassessment J. Mod. Opt. $23685-700$

[10] Vikram C and Billet M 1984 Far-field holography at non-image planes for size analysis of small particles Appl. Phys. B 33 149-53

[11] Fournier C, Barat C, Ducottet C and Fournel T 2003 Digital holography applied to PIV: hologram reconstruction and selection of a cloud of particles in the reconstructed volume 4th Pacific Symp. on Flow Visualization and Image Processing (Chamonix, France) pp 2-5

[12] Denis L, Fournier C, Fournel T, Ducottet C and Jeulin D 2006 Direct extraction of the mean particle size from a digital hologram Appl. Opt. 45 944-52

[13] Soulez F, Denis L, Thiébaut É, Fournier C and Goepfert C 2007 Inverse problem approach in particle digital holography: out-of-field particle detection made possible J. Opt. Soc. Am. A 24 3708-16

[14] Soulez F, Denis L, Fournier C, Thiébaut É and Goepfert C 2007 Inverse-problem approach for particle digital holography: accurate location based on local optimization J. Opt. Soc. Am. A 24 1164-71

[15] Gire J, Denis L, Fournier C, Thiebaut E, Soulez F and Ducottet C 2008 Digital holography of particles: benefits of the 'inverse problem' approach Meas. Sci. Technol. 1974005

[16] Goodman J W 2005 Introduction to Fourier Optics (Colorado: Roberts \& Co.)

[17] Lavrent'ev M and Shabat B 1958 Methods of the Theory of Functions of a Complex Variable (Moscow: Fizmatgiz) p 446

[18] Ooms T A 2008 Digital holographic particle image velocimetry Meas. Sci. Technol. 19074003 